(C) 2017 IEEE. Personal use of this material is permitted. Permission from IEEE must be obtained for all other uses, in any current or future media, including reprinting/republishing this material for advertising or promotional purposes, creating new collective works, for resale or redistribution to servers or lists, or reuse of any copyrighted component of this work in other works.

\title{
Energy Consumption and Reliability Performance of Survivable Passive Optical Converged Networks: Public ITS Case Study
}

\author{
E. Grigoreva, E. Wong, M. Furdek, L. Wosinska and C. Mas Machuca
}

\begin{abstract}
Access networks are evolving fast by increasing their capacity and coverage area, coping with a larger number of users and variety of terminals. Operators aim at keeping high network performance and quality of service but limiting their capital and operational expenditures by e.g., minimizing investments and energy consumption using power saving at the network components. To address these challenges this paper evaluates energy consumption, connection availability and failure detection time of three protection schemes applicable for converged access networks: Disjoint Fiber Protection, Energy-Efficient Disjoint Fiber Protection, and Reflective Disjoint Fiber Protection. The schemes are assessed by a case study considering public Intelligent Transport System (ITS). The studied ITS deploys a Dedicated Short Range Communications (DSRC) Radio Access Network connected to the service server through a protected passive access network. Comparison with unprotected architecture shows that the Reflective

Disjoint Fiber Protection offers low energy consumption and high connection availability, while it significantly reduces the failure detection time and hence, the connection interruption time.
\end{abstract}

Index Terms - Next generation optical access (NGOA), energy consumption, failure location

\section{INTRODUCTION}

A ccess networks are rapidly evolving to accommodate larger and more diverse types of terminals, services, bandwidth demands and higher reliability requirements. Further, advancements in the underlying technology are allowing for a decrease of components costs (e.g., colorless Optical Network Units (ONUs) [1]) and an increase of the passive reach. Consequently, operators are considering the migration of existing access networks (e.g., combining optical fiber and copper or using GPON) to new architectures in order to support potential new requirements (e.g. higher bandwidth, higher availability) but at limited cost.

One possible approach considered by operators is to deploy Hybrid Passive Optical Networks (HPONs) in their network domain. This architecture, as shown in Fig. 1, combines Wavelength Division Multiplexing (WDM) using wavelength demultiplexers such as Array Waveguides (AWG) with Time Division Multiplexing (TDM) based on
Power Splitters (PS). Such HPON architecture offers several advantages:

- Similar to GPON with Dynamic Bandwidth Allocation (DBA) but coping with higher bandwidth range (MBS get a full wavelength), HPON supports terminals with different bandwidth requirements, whereby terminals with lower bandwidth requirements, e.g., residential users, are connected to the PS whereas terminals with higher bandwidth requirements, e.g., Macro Base Stations (MBS), are connected directly to the AWGs.

- It can reuse any existing Optical Distribution Network (ODN) already deployed by the operator. For example, the HPON can reuse the ODN and the power splitter of an already deployed Gigabit Passive Optical Network (GPON).

- It allows greater maximum reach and hence, supports node consolidation with a reduced number of central offices. Node consolidation offers cost reductions through a lower number of active components, lower power consumption, less floor space, etc. [2].

- HPON can support a large client count which in turn depends on the splitting ratio of the AWG and the PS. Some possible examples as outlined in [3] combine AWGs of 40 and 80 wavelengths with 1:32 power splitters which results in more than 1200 and 2500 terminals, respectively.

HPON can serve as the underlying architecture to support converged access networks [4], whereby the same architecture can be used to interconnect different types of terminals (residential users, business users, MBSs, small cells, etc. based on their bandwidth requirements. To date, operators deploy different networks for different types of access clients, e.g., MBS and residential user are connected to different access networks. Having independent networks can be costly to install, deploy and maintain.

As noted earlier, aside from bandwidth, reliability performance is also increasingly important in the deployment of future access networks. The diversity of services and terminals could lead to higher connection availability requirements than that currently offered by existing unprotected access networks. One important concern of network operators is to find a compromise between the investments required to offer protected access and the expected increase of revenues from customers (i.e., how much are the customers willing to pay for increased network reliability performance. Different solutions have been proposed for HPON architectures, aiming to reduce the 
required investments [5], energy consumption and failure detection time [6].

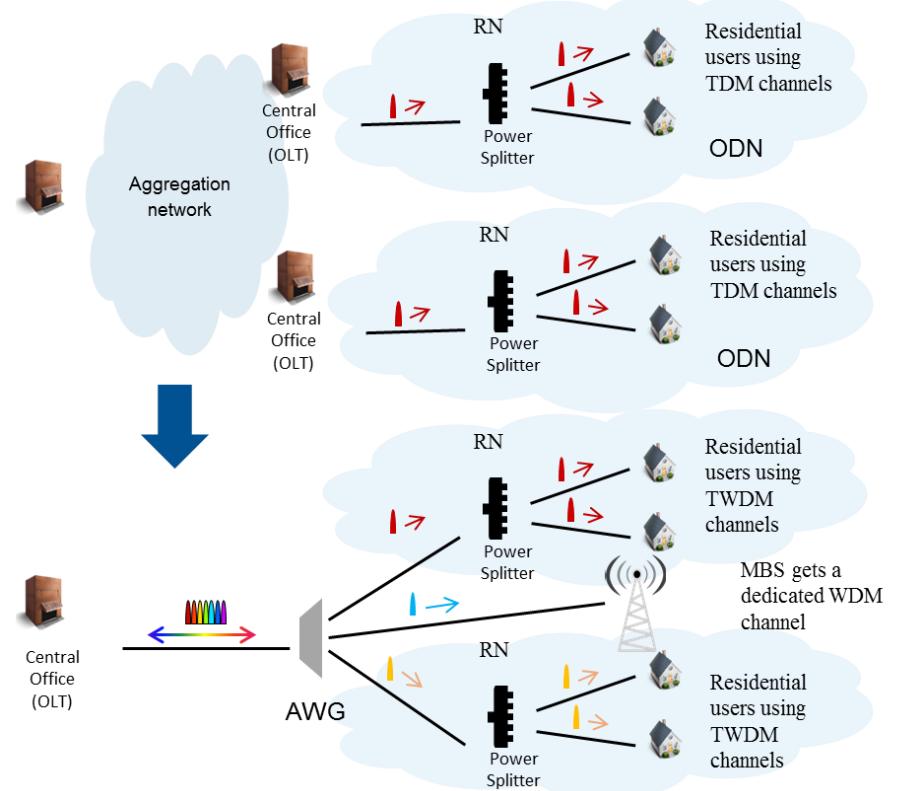

Fig. 1 Example of migration of a conventional power splitting access network (top of the figure) towards a Hybrid Passive Optical Network (HPON) solution (bottom of the figure)

The vast majority of optical access network studies are focused on telecom operators and their case studies to interconnect cabinets, MBSs, buildings, etc. In contrast, in this paper, we consider a different case study: Intelligent Transportation System (ITS). ITS was introduced in the early nineties and can be defined as a system in which information and communication technologies are applied to improve safety, transport efficiency, and provide information and entertainment. Unlike conventional communication systems, an ITS requires high reliability and low failure detection time for the road safety applications. Conversely, it is imperative that the survivability performance of any deployed ITS is critically studied. Generally, ITS is not bound to a specific technology [7]. The most prospective ITS technologies are Long Term Evolution (LTE) and IEEE 802.11p or Dedicated Short Range Communications (DSRC) [8]. To date, only some of the ITS services are realized with most of them yet to be implemented, especially those relating to safety applications. For the intended implementations in some countries like in USA [9], DSRC was chosen as the main technology for ITS realization.

In this paper, we apply HPON protection schemes to the ITS infrastructure based on the DSRC Radio Access Network (RAN). To narrow the scope of ITS, we explore the applicability of three HPON protection schemes to onground public transport, such as the bus, tram and trolleybus lines.

The focus of our case study lies in the backhaul infrastructure provisioning for DSRC-based RAN. Fig. 2 presents the scenario under investigation. The backhaul connects DSRC base stations or Road Side Units (RSUs) to the Service Server (SS) for centralized processing and control. As the maximal data rate supported by DSRC RSUs is only up to $54 \mathrm{Mbps}$ [10], GPON can be used for the backhaul.
In our setup, Service Server (SS) is collocated with the OLT, at the central office. The fiber between the OLT and Power Splitter (PS) is referred to as Feeder Fiber (FF). The fiber between PS and ONU is Last Mile Fiber (LMF). All ONUs are collocated with the respective RSUs.

The tree topology of the network with no redundant links makes the ITS vulnerable to link cuts which, depending on the particular location of the cut, may leave a great number of RSUs unconnected and jeopardizing the effectiveness of the ITS. In order to ensure connectivity in the presence of link cuts, a protection scheme must be deployed, taking into consideration various trade-offs between the different schemes.

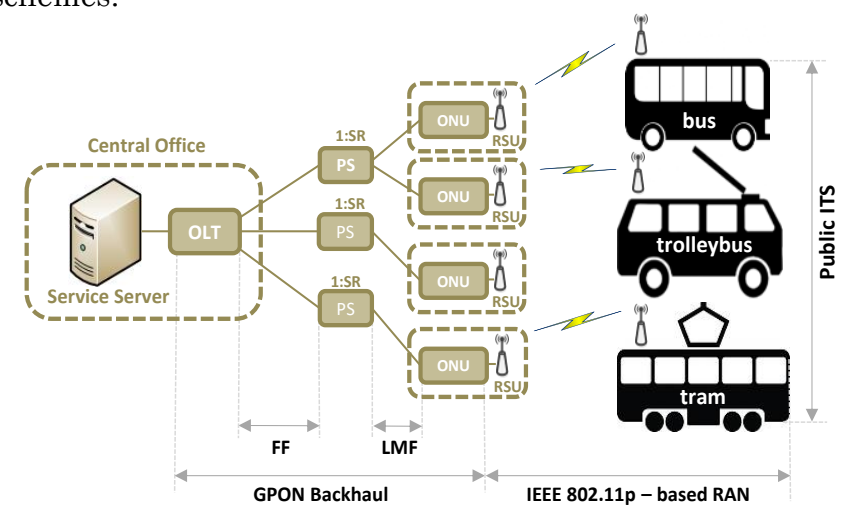

Fig. 2 Communication Network Infrastructure for public ITS with DSRC-based RAN and GPON backhaul

This paper aims at comparing power consumption, connection availability and failure detection times of three different protection schemes for HPON architectures, applied to a particular case study of ITS. The details of these three protection schemes are presented in Section II. In Section III, the ITS case study and the considered network including the user activity, network planning, and evaluation methodology, are described. Evaluation results are then presented in Section IV. Finally, the main conclusions are drawn and summarized in Section V.

\section{Protection Schemes}

Due to the high bandwidth and connection availability required by RSUs, this paper aims at comparing different solutions to provide protection to RSUs. Note that each RSU is collocated with its corresponding ONU. In that respect, three HPON protection alternatives proposed in [6] are presented here and compared against the unprotected HPON architecture (refer to Fig. 1) in terms of energy consumption, connection availability and failure detection time. The architectures are termed: (a) disjoint fiber protection HPON (denoted as P-Active); (b) energy-efficient disjoint fiber protection HPON (denoted as P-A\&S); and (c) reflective disjoint fiber protection HPON (denoted as RP). Note that in the descriptions of the studied architectures, a two stage network with two Remote Nodes (RNs) is considered to reflect practical deployment scenarios where RN1 implements the wavelength de/multiplexing function and RN2 implements the power splitting function. Also note that, in order to achieve protection and survivability, active components are added only to CO and RSUs, leaving the optical distribution network completely passive to allow reuse of legacy TDM-PONs for HPON deployments. The three protected HPON architectures considered in this work are described as follows. 


\section{A. Disjoint Fiber Protection (P-Active)}

The disjoint fiber protection HPON architecture (denoted as P-Active) is a protection scheme whereby both the Feeder Fiber (FF) and Last Mile Fiber (LMF) of each ONU are protected. As illustrated in Fig. 3, this architecture provides 1:1 protection where each RSU is connected to the $\mathrm{CO}$ via a disjoint $\mathrm{FF}$ and a disjoint $\mathrm{DF}$. Here, the standard definition of disjointness is considered whereby disjoint fibers are those located in different geographically separated ducts. In contrast to the unprotected HPON architecture, redundant and additional components/ equipment are deployed at the $\mathrm{CO}, \mathrm{RN}$, and ONU to achieve protection. At the $\mathrm{CO}$ and $\mathrm{ONU}$, an additional optical switch denoted as OSW1 and OSW2 in the figure, respectively, is implemented for protection switching. At RN1, two $1 \times 2$ couplers and two $2 \times \mathrm{N}$ AWGs are deployed.

Under normal operating conditions, OSW1 and OSW2 are in BAR state with upstream and downstream traffic traversing the primary $\mathrm{FF}$ and the primary $\mathrm{DF}$. When a failure (primary FF, primary DF, $1 \times 2$ coupler, or AWG) occurs, a Loss of Signal (LOS) is detected at the CO and the RSU, which triggers OSW1 and OSW2 to change to the CROSS state and shift the traversing signals onto the disjoint protection paths.

\section{B. Energy-Efficient Disjoint Fiber Protection (P-A\&S)}

The Energy-efficient Disjoint Fiber Protection scheme (denoted as P-A\&S) has the same architecture as the PActive architecture but as alluded to by its name, this protection HPON architecture achieves high energyefficiency due to the sleep state capability of its ONUs (integrated with RSUs in this study) [11]. In this architecture, ONUs can transition between an active and a sleep state where the latter supports powering down transceivers to save energy when no data is to be transmitted/received, i.e. during quiet period. The drawback of this scheme is that failures that occur during the quiet period will be detected only when the ONU transitions back to the active state. Hence, the outage duration and, consequently, the penalty on operators will be larger than in $\mathrm{P}$-Active albeit P-A\&S is more energy-efficient.

Another drawback of the P-A\&S architecture is that the SS, which relies on LOS of upstream transmissions to detect failures in the network, may erroneously trigger protection switching of OSW1 when all ONUs are in a sleep state. Loss-of-Signal is commonly used in conventional networks to detect the occurrence of an equipment/fiber failure and to trigger protection switching of the affected traffic onto the backup path. A LOS alarm will be activated at the headend if an incoming signal has no transitions over a period of $175 \pm 75$ contiguous pulse intervals (ITU-T G.775 [12]). Using the absence of incoming transmissions to detect failures and trigger LOS alarm is therefore ineffective in P-A\&S since (a) ONUs in sleep state have zero output power and may erroneously set off the LOS alarm at the $\mathrm{CO}$ and (b) an ONU in sleep state cannot continuously monitor the network until it transitions out of the sleep state to reinstate LOS monitoring.

\section{Reflective Disjoint Fiber Protection (RP)}

The Reflective Disjoint Fiber Protection (denoted as RP) addresses the limitations of the P-A\&S by employing an outof-band continuous wave monitoring signal, $\lambda_{M}[6]$. Here, $\lambda_{M}$ is spaced an integer multiple of the AWG's free spectral range away from the ONU's downstream and upstream wavelength channels. Such wavelength assignment allows $\lambda_{\mathrm{M}}$ to be detected at and reflected from the ONU.

The RP architecture, depicted in Fig. 4, achieves the energy efficiency of the P-A\&S scheme by allowing sleep state in quiet periods, while it also enables fast failure detection. Compared to an unprotected HPON, this architecture requires additional equipment to facilitate protection. This includes an optical switch (OSW1), two WDM filters, and a monitoring module at the CO; two $1 \times 3$ couplers and two $2 \times \mathrm{N}$ AWGs at RN1; and two WDM filters, an optical switch (OSW2), and a monitoring receiver (MON RX) at each ONU. Under normal working conditions, $\lambda_{\mathrm{M}}$ is launched into the ODN and a fraction of the optical power of $\lambda_{\mathrm{M}}$ is reflected at the AWG. The reflected $\lambda_{\mathrm{M}}$ is then detected back at the monitoring module at the $\mathrm{CO}$.

In the event of a working path failure, the reflected $\lambda_{\mathrm{M}}$ is absent at the OLT thereby triggering the transition of OSW1 into CROSS state. Also, the absence of $\lambda_{\mathrm{M}}$ at the ONU triggers the transition of OSW2 into CROSS state and traffic is sent through the protection LMF. This RP architecture does not rely on upstream transmissions to trigger LOS alarm. Consequently, failures can be continuously monitored during quiet period when ONUs are in the sleep state.

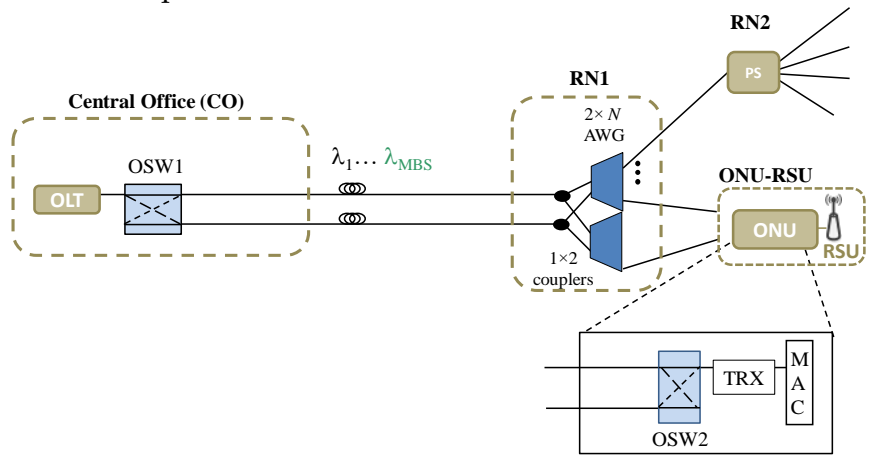

Fig. 3 Disjoint Fiber Protection (P-Active) and Energy-Efficient Disjoint Fiber Protection (P-A\&S) HPON architecture

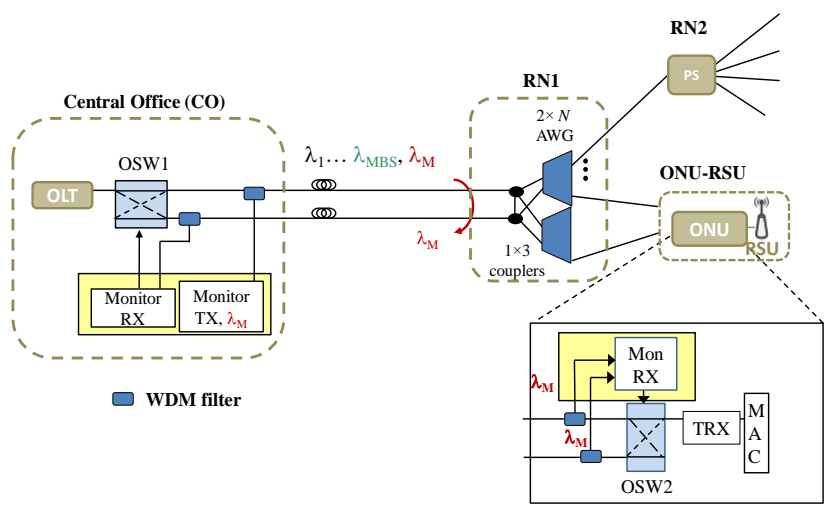

Fig. 4 Reflective Disjoint Fiber Protection (RP) HPON architecture

\section{PUBlic ITS CASE STUdY}

A first comparative study of energy consumption, connection availability, failure detection time and restoration time in the presence of failure was carried out in [13], considering residential users and LTE MBS for different areas given average traffic models available in literature. In this paper, we consider a real case study of a public ITS aiming at providing services for buses, trams and 
trolleybus in the central area in Munich (Germany) of approximately $7 \mathrm{~km}^{2}$.

ITS research normally focuses on the technology applicability, while neglecting the infrastructure implications, e.g., [10]. The implications, on network planning, protection and power consumption are significant. The public ITS network is more sparse than FTTB, requires higher reliability, but at the same time can be less bandwidth-demanding compared to residential users. As ITS is planned for implementation, for example USA has decided on DSRC-based ITS implementation [14], it is crucial to understand the aforementioned factors that differ from conventional networks.

In this section, we introduce the case study methodology. We define the scenario, user activity patterns and explain network planning concepts. Finally, we present the parameters and equations for power and availability calculations.

\section{A. User RSU Activity Description}

An advantage of focusing on public ITS is the availability of information regarding the activity times of public transport. Fig. 2 illustrates the used case study scenario, which we use in this work evaluation. This scenario was first introduced in [15]. The RSU and thus the ONU placement is fixed, that is, the placement is based on offering coverage along all considered routed ITS, e.g. day and night busses. In our study, based on DSRC radio access, the inter-station distance between the RSUs is $500 \mathrm{~m}$ due to communication range limitations of DSRC [16]. Such placement results in 38 RSUs and corresponding ONUs.

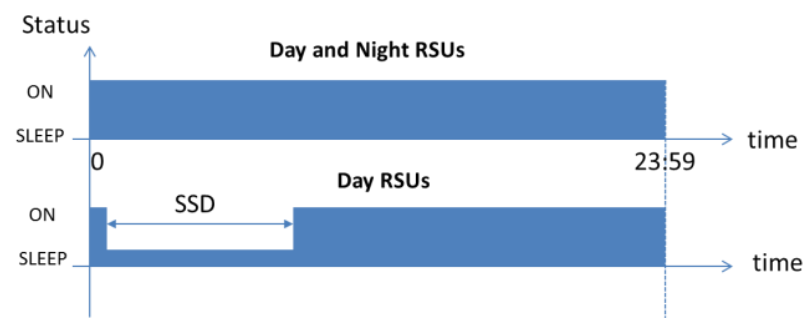

Fig. 5 Sleep Slot Duration (SSD) for public ITS

TABLE I

RSU ACTIVITIES BASED ON ON-GROUND PUBLIC TRANSPORT ACTIVITY

\begin{tabular}{|c|c|}
\hline Day RSUs, id & Day and Night RSUs, id \\
\hline $\begin{array}{c}3,4,12,13,16,17,19,20,25,26 \\
27,28,29,30,31,33,35,36\end{array}$ & $\begin{array}{c}1,2,5,6,7,8,9,10,11,14,15 \\
18,21,22,23,24,32,37,38\end{array}$ \\
\hline $\begin{array}{l}\text { Min. SSD } \\
\text { 3h10m }\end{array}$ & Always active \\
\hline
\end{tabular}

For the RSUs that are active only during the day, we predetermine its sleep duration by considering the time slot when all RSUs are not used, that is, the time when no transport (i.e., no buses, trams nor trolleybus) circulate through the area covered by the RSU. Fig. 5 illustrates the physical meaning of SSD. As timetables are different for working days, weekends and holidays, we consider a minimal and a maximal SSD. Table I provides details of the two groups of RSUs, day RSUs and day and night RSUs. The minimal SSD and RSU allocation to day or day and night was obtained from route timetables ${ }^{1}$ that are publically available [17]. The maximal SSD was arbitrarily chosen for comparison purposes. This value can be seen as an upper bound for the sensitivity analysis or a maximum time, representing weekend, holiday, other city and other timetable differences.

\section{B. Network planning}

Once a set of RSUs and their activities are defined, we first need to plan the network, i.e., define the interconnections between the RSUs (ONUs), PSs and CO. In our case study, network planning consists of two parts: RSU clustering and fiber routing. RSU clustering can be done independently from the user activity pattern, i.e., based solely on geographic position with the objective to minimize the overall fiber length. This option is referred to as "ind". Alternatively, the RSUs can be grouped depending on the activity pattern, i.e., clustering the day RSUs and the day and night RSUs into separate clusters. In this case, it is possible to put some OLT ports to sleep if all RSUs (and ONUs) belonging to the PS connected to an OLT port are put to sleep. This approach is referred to as "dep" and it has potential to save more energy.

We further explore two RSU clustering scenarios: greenfield and brownfield. In greenfield scenario there is no prior infrastructure available. We investigate this setting using PSs with different Splitting Ratios (SR): 8, 16 and 32 considering an $80 \%$ port use (the remaining unused $20 \%$ of ports are kept for redundancy or future use [5]). In brownfield scenario there is an existing GPON infrastructure for FTTB with SR $=32$ and $80 \%$ port use. This leaves us with seven ports at the PS that are free for ITS implementation.

For fiber routing, we use the shortest path algorithm in street metric, thus yielding a geographical planning approach. The maps used for our case study are Open Street Maps [18]. Routing scripts and all the geography related processing was performed using ArcGIS [19].

TABLE II

GPON COMPONENT PARAMETERS [21]

\begin{tabular}{|c|c|c|c|c|c|}
\hline Element & \multicolumn{3}{|c|}{ Power, W } & \multicolumn{2}{|c|}{ Availability } \\
\hline \multicolumn{6}{|c|}{ Active Components } \\
\hline \multirow{2}{*}{$\begin{array}{l}O L T \text { per } \\
\text { port }^{1}\end{array}$} & active & 13 & $P_{O L T \text { port }}^{\text {active }}$ & \multirow{2}{*}{0.9999638} & \multirow[b]{2}{*}{$a_{O L T}$} \\
\hline & sleep ${ }^{2}$ & 3.9 & $P_{\text {OLT }}^{\text {sleep }}$ & & \\
\hline \multirow{2}{*}{$O N U$} & active & 5 & $P_{O N U}^{\text {active }}$ & \multirow{2}{*}{0.999961} & \multirow{2}{*}{$a_{O N L}$} \\
\hline & sleep & 0.75 & $P_{O N U}^{\text {sleep }}$ & & \\
\hline$O S$ & \multicolumn{2}{|c|}{1} & $P_{O S}$ & 0.999994 & \multirow{3}{*}{$a_{O S}$} \\
\hline TxMon & \multicolumn{2}{|c|}{1} & $P_{T X \_m o n}$ & $(0.9999994)^{3}$ & \\
\hline RxMon & \multicolumn{2}{|c|}{1} & $P_{R X \_ \text {mon }}$ & $(0.9999994)^{3}$ & \\
\hline \multicolumn{6}{|c|}{ Passive Components } \\
\hline PS & \multicolumn{2}{|c|}{-} & - & 0.999999 & $a_{P S}$ \\
\hline Coupler & \multicolumn{2}{|c|}{-} & - & 0.9999993 & $a_{c}$ \\
\hline WDM filter & \multicolumn{2}{|c|}{-} & - & 0.999994 & $a_{f}$ \\
\hline Fiber per $\mathrm{km}$ & \multicolumn{2}{|c|}{-} & - & 0.9999857 & $a_{k m}$ \\
\hline
\end{tabular}

\section{Power and Connection Availability}

In this subsection, we describe the methodology used in evaluating the power and connection availability of the three protection HPON architectures. We present the yearly 
power consumption for the active components of the GPON backhaul network. We use graphical representation of Reliability Block Diagrams (RBDs) to describe equations for connection availability. All calculations are carried out for the four cases of HPONs: unprotected (UP), P-Active, PA\&S and RP.

Table II summarizes the GPON component parameters. Although GPON is a passive network, the transceivers in the $\mathrm{CO}$ and ONU are active and thus consume energy. At the CO, each OLT port can transition to sleep mode if all connected ONUs are in the sleep mode.

A port in sleep mode consumes only $30 \%$ of the power consumed in the active state [23]. Here the Layer 2 optical equipment, i.e., switching, is excluded from power consumption calculations. It is important to point out that the values were obtained by only considering the physical layer OLT power consumption. For the protection case, further active elements are added, for example Optical Switches (OSs), monitoring transmitters (TxMons) and receiver (RxMons).

Power. First, we define power consumption for the unprotected and P-Active protection architectures. The active components in this case are never put to sleep. The total energy consumption per year can be then calculated as:

$P^{U P}=\left(P_{O L T \text { port }}^{\text {active }} \cdot N_{P S}+P_{O N U}^{\text {active }} \cdot N_{O N U}\right) \cdot 24 \cdot \frac{365}{1000}$

$P^{P-\text { Active }}=\left(P^{U P}+P_{O S} \cdot\left(N_{P S}+N_{O N U}\right)\right) \cdot 24 \cdot \frac{365}{1000}$

where $\mathrm{N}_{\mathrm{PS}}$ and $\mathrm{N}_{\mathrm{ONU}}$ are the numbers of PSs and ONUs, respectively. Further, we adopt the notation of $\mathrm{N}_{\text {element }}$ to denote the number of any network element.

In the unprotected case, the active components are the OLT ports and ONUs, as represented by Eqn. 1. For the PActive protection case, Eqn. 2, we add one OS for the OLT and an OS per ONU to provide protection. Thus, consumed energy is determined by the number of PSs and ONUs.

In P-A\&S and RP, equipment can be put to sleep to save power. An ONU can transition to sleep outside activity hours. In our case, the RSUs and their corresponding ONUs that are active during the day can be put to sleep during SSD. However, an OLT port can be put to sleep only if all the ONUs connected to the same PS are in sleep mode. If during assignment of ONUs to PSs, we do not take into account user activity patterns, the OLT ports will, in most of the cases, not be transitioned to sleep. The power consumption for P-A\&S are described as follows:

$$
\begin{aligned}
P_{\text {ind }}^{P-A \& S}= & \left(P_{\left(24-T_{S S D}\right)}^{\text {ind }(P-A \& S)}+P_{T_{S S D}}^{\text {ind }(P-A \& S)}+P_{24}^{\text {ind }(P-A \& S)}\right) . \\
& \cdot \frac{365}{1000},
\end{aligned}
$$

whereby the three terms on the Right Hand Side (RHS) represent the power consumption of the network components that are active during the various intervals of the day. The first term represents power consumption in the time interval $\left(24-\mathrm{T}_{\mathrm{SSD}}\right)$, in which all ONUs are active (day):

$P_{\left(24-T_{S S D}\right)}^{\text {ind }(P-A \& S)}=P_{O N U}^{\text {active }} \cdot N_{O N U} \cdot\left(24-T_{S S D}\right)$.

The second term represents power consumption of the $\mathrm{T}_{\mathrm{SSD}}$, i.e., sleep time (SSD) or night, in which the day ONUs are in a sleep mode and only the night ONUs are active:

$P_{T_{S S D}}^{\text {ind }(P-A \& S)}=\left(P_{O N U}^{\text {sleep }} \cdot N_{O N U}^{\text {day }}+P_{O N U}^{\text {active }} \cdot N_{O N U}^{\text {night }}\right) \cdot T_{S S D}$
Finally, the last term of the RHS represents power consumption of the OS and OLT ports that are always active:

$P_{24}^{\text {ind }(P-A \& S)}=\left(\left(N_{P S}+N_{O N U}\right) \cdot P_{O S}+P_{O L T_{\text {port }}}^{\text {active }} \cdot N_{P S}\right) \cdot 24$

For RP, the general equation of its power consumption is the same as (3) but with the additional power contributions from active monitoring components. Hence:

$$
\begin{aligned}
P_{24}^{\text {ind }(R P)}=\left(\left(N_{P S}+N_{O N U}\right) \cdot P_{O S}+\left(P_{T X_{-} \text {mon }}+P_{R X_{m o n}}\right) \cdot\right. \\
\left.\cdot N_{P S}^{\text {day }}+P_{R X_{-} m o n} \cdot N_{O N U}^{\text {day }}+P_{O L T_{\text {port }}}^{\text {active }} \cdot N_{P S}\right) \cdot 24
\end{aligned}
$$

As discussed in Section III.B, RSUs can be grouped depending (dep) on the activity pattern. In this case, some OLT ports can be transitioned to sleep mode during the $T_{S S D}$. The number of such ports corresponds to the number of PSs, for which all connected ONUs can be simultaneously transferred to the sleep mode. In our case, all day ONUs can be put to sleep during $T_{S S D}$. Power consumption in $T_{S S D}$. interval is then composed of active and sleep parts. The active part is due to active (always-on) ONUs and their corresponding OLT ports. The sleep part, respectively, is due to day ONUs and their corresponding OLT ports:

$$
\begin{aligned}
P_{T_{S S D}}^{\text {dep }(P-A \& S)}= & \left(P_{O N U}^{\text {active }} \cdot N_{\text {ONU }}^{\text {night }}+P_{\text {OLT }}^{\text {active }} \cdot N_{P S}^{\text {night }}+\right. \\
& \left.+P_{\text {ONU }}^{\text {sleep }} \cdot N_{\text {ONU }}^{\text {day }}+P_{\text {OLT }}^{\text {sleep }} \cdot N_{P S}^{\text {day }}\right) \cdot T_{S S D}
\end{aligned}
$$

During the time $\left(24-T_{S S D}\right)$, all the ONUs and thus OLTports are active. The power consumption in the time interval $24-T_{S S D}$ can be calculated as:

$$
\begin{aligned}
& P_{\left(24-T_{S S D}\right)}^{\text {dep }(P-A \& S)}=\left(P_{O L T_{p o r t}}^{\text {active }} \cdot N_{P S}+P_{O N U}^{a c t i v e} \cdot N_{O N U}\right) . \\
& \cdot\left(24-T_{S S D}\right) \text {. }
\end{aligned}
$$

Finally, OSs are active 24h:

$P_{24}^{\text {dep(P-A\&S) }}=P_{O S} \cdot\left(N_{P S}+N_{O N U}\right) \cdot 24$.

RP power consumption differs from the P-A\&S only in the $24 \mathrm{~h}$ power component due to the presence of the monitoring system and is given by:

$$
\begin{aligned}
P_{24}^{\text {dep }(R P)}= & \left(P_{O S} \cdot\left(N_{P S}+N_{O N U}^{\text {day }}\right)+\left(P_{T X_{m o n}}+P_{R X_{m o n}}\right) .\right. \\
& \left.\cdot N_{P S}^{d a y}+P_{R X_{m o n}} \cdot N_{O N U}^{d a y}\right) \cdot 24 .
\end{aligned}
$$

Connection availability is defined as the probability that a connection is in an operable state at a random point of time. The commonly used availability model is graphically illustrated by Reliability Block Diagram (RBD) [20], where blocks represent components which are connected in series and in parallel from a reliability point of view. The availability of each component $i$ is denoted by $a_{i}$, see Table II. The calculation takes into account the average fiber length. We define average FF availability as $a_{F F}=a_{k m} l_{F F}$, where $l_{F F}$ is the average FF length in $\mathrm{km}$. We define LMF availability, $a_{L M F}$, in the same way. Protection elements are labelled following the working path notation, but with a dash, e.g., $a_{F F}$, .

The RBD for the UnProtected (UP) architecture is shown in Fig. 6. The RBD consists of the blocks representing OLT, FF, PS, LMF and ONU connected in series and hence, the availability, denoted by $\mathrm{a}_{\text {con }}^{\mathrm{UP}}$, can be computed as the product of their availabilities (Eqn. 12). 
$a_{\text {con }}^{U P}=a_{O L T} \cdot a_{F F} \cdot a_{P S} \cdot a_{L M F} \cdot a_{O N U}$

(12)

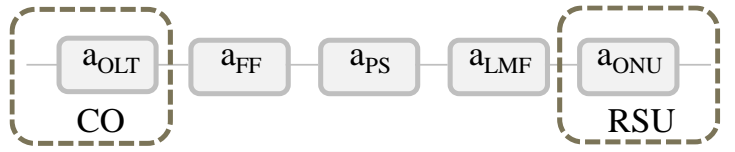

Fig. 6 RBD of the UnProtected (UP) architecture

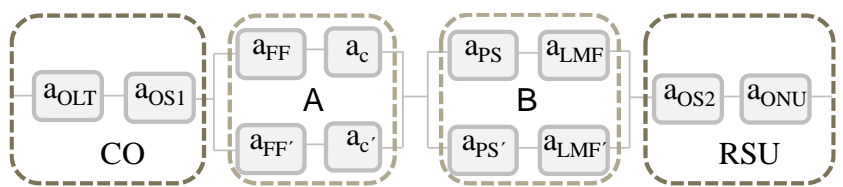

Fig. 7 RBD of the P-Active or P-A\&S architectures

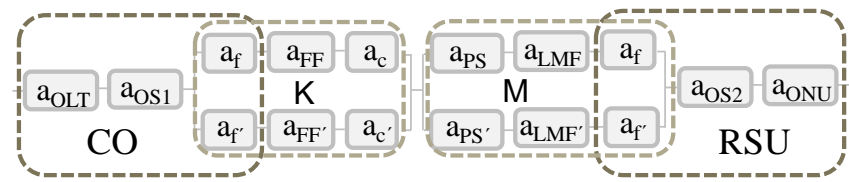

Fig. 8 RBD of the RP architecture

Having the same network components, P-Active and $\mathrm{P}$ A\&S feature the same RBDs, shown in Fig. 7, and availability calculation, expressed by Eqn. 13, as the network components are the same. Here $a_{c}$ stands for the availability of the coupler.

$a_{\text {con }}=a_{O L T} \cdot a_{O S 1} \cdot a_{A} \cdot a_{B} \cdot a_{O S 2} \cdot a_{O N U}$

$a_{A}=1-\left(1-a_{F F} \cdot a_{c}\right)\left(1-a_{F F^{\prime}} \cdot a_{c^{\prime}}\right)$

$a_{B}=1-\left(1-a_{P S} \cdot a_{L M F}\right)\left(1-a_{P S^{\prime}} \cdot a_{L M F^{\prime}}\right)$

For the RP architecture, we have additional filter components $\left(\mathrm{a}_{\mathrm{f}}\right)$ that influence connection availability model, as shown in Fig. 8. The RP connection availability can be calculated as follows:

$a_{\text {con }}=a_{O L T} \cdot a_{O S 1} \cdot a_{K} \cdot a_{M} \cdot a_{O S 2} \cdot a_{O N U}$,

$a_{K}=1-\left(1-a_{f} \cdot a_{F F} \cdot a_{c}\right)\left(1-a_{f^{\prime}} \cdot a_{F F^{\prime}} \cdot a_{c^{\prime}}\right)$,

$a_{M}=1-\left(1-a_{P S} \cdot a_{L M F} \cdot a_{f}\right)\left(1-a_{P S^{\prime}} \cdot a_{L M F^{\prime}} \cdot a_{f^{\prime}}\right)$.

\section{CASE Study Results}

In this section, we present the case study results on the power consumption and connection availability for the greenfield and brownfield case. We also show a sensitivity analysis of the power consumption to the proportion of the day and night ONUs.

Power consumption figures in this Section show independent and dependent clustering with minimum and maximum SSD. For example, notation "ind_ssd3.1" would stand for the results for independent clustering with SSD = 3.1 hours. For the brownfield scenario, additional notation of "+b" is used, it means that in this case residential users were taken into account, i.e., existing FTTB network.

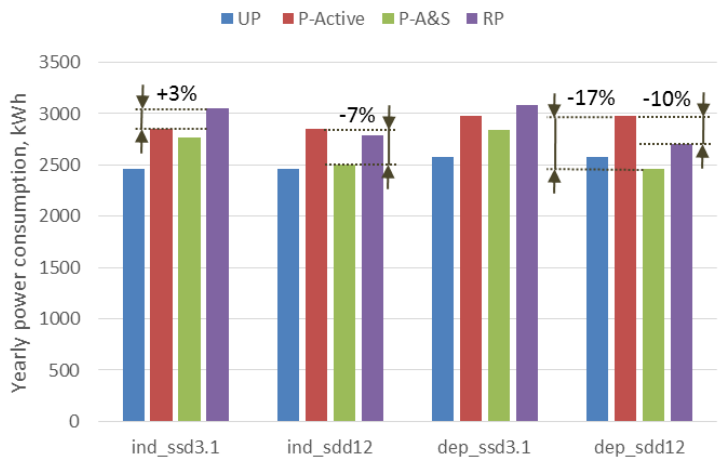

Fig. 9 Yearly network power consumption, $\mathrm{SR}=8$

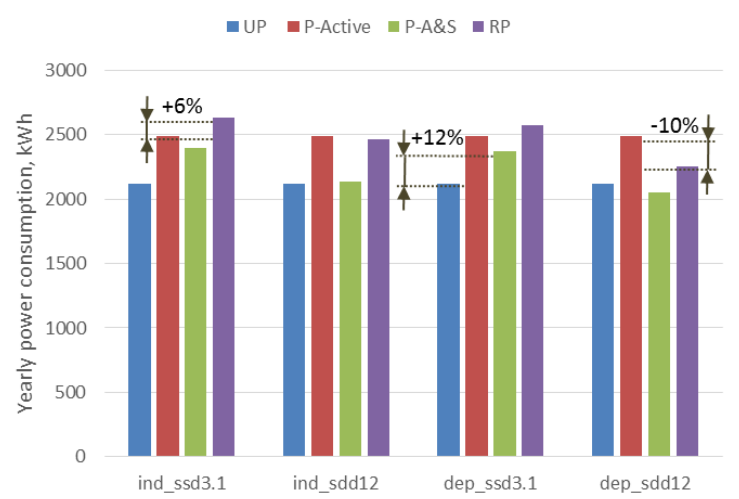

Fig. 10 Yearly network power consumption, SR=16

Figs. 9 and 10 depict power consumption per year for SR $=8$ and $\mathrm{SR}=16$, respectively, showing the outcomes arising from the independent and dependent ONU (or equivalently RSU) grouping for two different sleep slot duration values. The power consumption is the lowest for the unprotected case (UP) as it has the lowest number of active components, i.e., only OLT ports and ONUs. In the case of independent RSU clustering, there are in total seven PSs with $\mathrm{SR}=8$. For the activity pattern case- dependent clustering, there are four clusters for day ONUs and four clusters for day and night ONUs. This leads to a total of eight PSs, one more than the independent clustering. It can be observed that the power consumed by the additional active equipment due to an extra cluster, and thus by the extra OLT port, is compensated by the energy saving of the P-A\&S and RP schemes.

For P-Active there are several additional active components such as OSs that are always active. Consequently, changing SSD does not impact the power consumption. As for P-A\&S, activity information of the public ITS is exploited to place some of the active components into sleep mode during SSD. Unsurprisingly, power consumption of P-A\&S is reduced by up to $7 \%$ compared to P-Active, where savings are higher for longer SSD. The number of PSs derives the number of additional active elements, and the SSD has to be long enough to compensate for the increase in the power consumption. For example, with $\mathrm{SR}=8$ dependent clustering results in more clusters, i.e., PSs, and SSD $=3.1$ hours results in more power consumption than in the independent case, while SSD $=12$ hours, in lower power consumption of the dependent clustering.

Finally, the power consumption of the RP architecture fluctuates around the P-Active values. Specifically, with $\mathrm{SSD}_{\min }$ it is $3 \%$ higher and with $\mathrm{SSD}_{\max }$ it is $10 \%$ lower. This small additional power consumption is attributed to the additional monitoring components but this is in exchange

TABLE III

FAILURE DETECTION AND RESTORATION TIMES

\begin{tabular}{ccccc}
\hline \hline$[\mathrm{ms}]$ & UP & P-Active & P-A\&S & RP \\
\hline Detection & 3.5 & 3.5 & 3.5 & 0.000524 \\
Restoration & $14400000^{1}$ & $10.5^{2}$ & $10.5^{2}$ & $10.5^{2}$ \\
\hline
\end{tabular}

1 This value represents a four hour reparation time [26], including the traveling time to the failure site, testing and independent of the number of required technicians.

2 Switching time to the protection path. 
for significantly improved failure detection times.

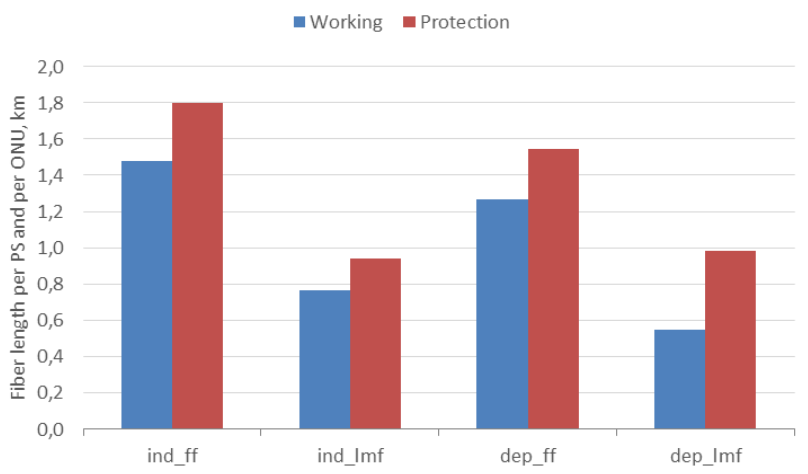

Fig. 9 Fiber length with independent and dependent clustering, normalized to the number of PSs for FF and ONUs for LMF. SR=16

Fig. 10 shows that with the same amount of PSs (number of clusters) the activity pattern dependent ONU clustering results in the same power consumption levels for UP and PActive. As for P-A\&S, the additional components result in up to $12 \%$ additional power consumption compared to the unprotected case. As for the RP architecture, its yearly power consumption shows similar fluctuations as in the previous case. One important deployment consideration when deciding upon whether to plan for independent or dependent clustering is that the former requires longer fiber, as shown in Fig. 11. Here, we compare the fiber length of independent and dependent clustering, normalized to the number of PSs for FF and ONUs for LMF. How much additional fiber is required in the dependent clustering over the independent clustering depends on the topology connectivity and ONU (RSU) density. In our case, the topology is well connected, but the ONU density is low (compared to a typical FTTB scenario). The total additional fiber length is only about $11 \%$.

Fig.12 illustrates connection availability. From the unprotected case (UP), we can clearly see the impact of clustering and thus fiber lengths. That is the connection availability for the dependent clustering is always lower than that for an independent one. As expected, all protection schemes improve connection availability, leveraging the difference between clustering and SRs. Most importantly, the results indicate the similar reliability performance of the protection schemes. Consequently, the decisive performance metric then comes down to the failure detection time.

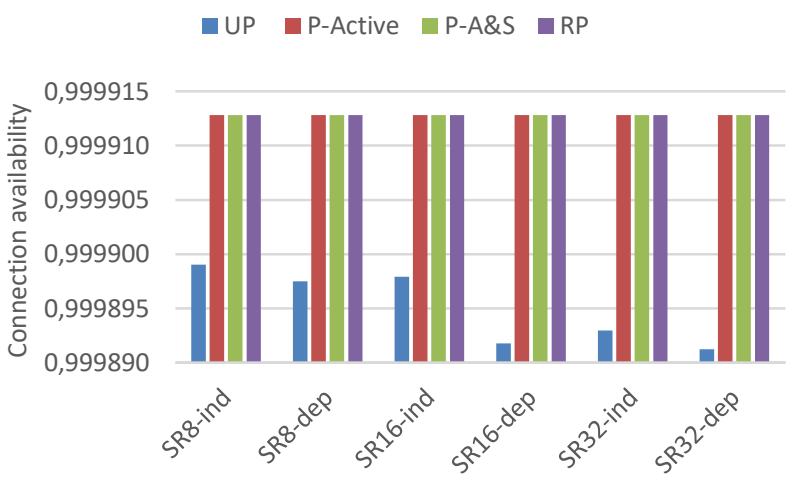

Fig. 12 Connection availability for the greenfield scenario
For ITS safety applications, which is the primary goal of most implementations, low failure detection time is a crucial metric. For example, for pre-crash sensing warning, the maximum latency should be as low as $50 \mathrm{~ms}$, and a standard safety application latency is bounded by $100 \mathrm{~ms}$ [18]. In the case of a failure, the detection and restoration time shall be as low as possible.

Table III summarizes the failure detection and restoration times for all the protection architectures and compares their values to the unprotected architecture [21], [22]. So the RP architecture is able to provide the lowest failure detection time, thus minimizing the delay due to the failure.

- $75 \%$ day ONUs $\quad 25 \%$ day ONUs

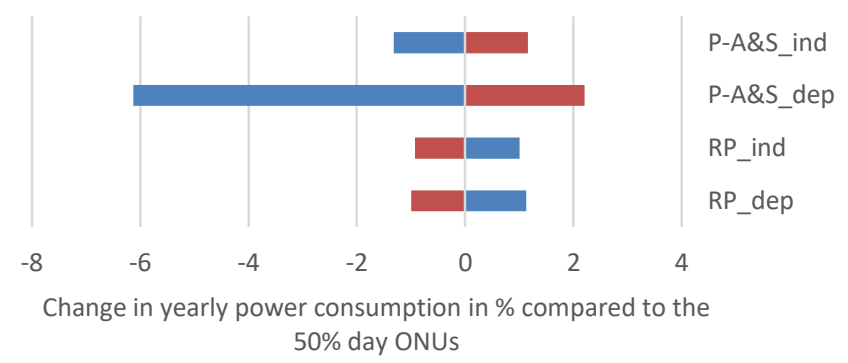

Fig. 13 Yearly power consumption sensitivity analysis, $\mathrm{SR}=16$. We compare $50 \%$ of day ONU with an increase and decrease by $25 \%$ showing the difference between power consumption with min SSD

$75 \%$ day ONUs $\quad 25 \%$ day ONUs

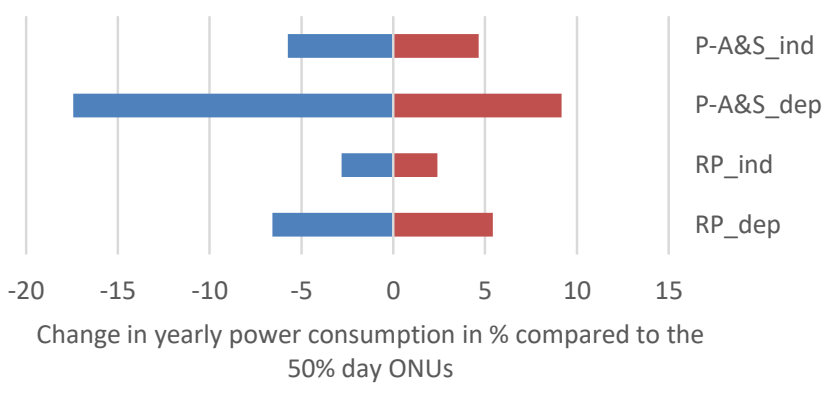

Fig. 14 Yearly power consumption sensitivity analysis, $\mathrm{SR}=16$. We compare $50 \%$ of day ONU with an increase and decrease by $25 \%$ showing the difference between power consumption with max SSD

Finally, Figs. 13 and 14 show the sensitivity analysis of the yearly power consumption for different portions of dayonly ONUs (increased or decreased by $25 \%$ ) with minimal $\mathrm{SSD}=3 \mathrm{~h} 10 \mathrm{~m}$ (Fig. 12) and maximal SSD $=12 \mathrm{~h}$ (Fig. 13). P. A\&S shows the same trends for both values of SSD. Power consumption has an inverse relation with the number of day ONUs. The more day ONUs there are, the more can be put to sleep. As there are no additional active components, compared to the P-Active, this directly results in power consumption decrease. As for the RP, it can be observed in Fig. 13 that an increase in the number of day ONUs results in power increase. This effect is due to the addition of active monitoring components and the gain from the sleep mode being insufficient to compensate for the power consumption increase. Fig.14 shows the opposite case, when the chosen SSD is adequate, and hence the reduction in power consumption from sleeping ONUs outweighs the additional 
power consumption from additional active monitoring components.

\section{B. Brownfield}

The main particularity of the brownfield scenario is that there is existing infrastructure, e.g., FTTB that should be exploited for ITS deployment. As it has already been mentioned, when planning a network, $20 \%$ of the RN ports are left for future use. In this brownfield scenario, any of the available $20 \%$ of the ports can be used to interconnect the RSU ONUs with the SS at the OLT.

In a brownfield case study, we assume an existing FTTB GPON network for 4877 buildings, clustered with SR $=32$ and $80 \%$ port use. This leaves us with seven free ports per installed PS. The FF in this case is already installed for the FTTB. For the ITS backhaul, we reuse this GPON network and cluster the ONUs to already available PSs. This clustering, as in the greenfield, is done either independently from or dependently on user activity patterns. In activitydependent clustering, we first cluster the RSUs that are active only during the day and connect them to the closest PS, which was already placed according to the FTTB planning. The PSs to which the day ONUs are connected, with no more ports available, are then excluded from the possible PSs for the day and night RSUs in the second stage. In the greenfield scenario, there was no need for PS exclusion as all PSs are newly placed, depending on the network needs.

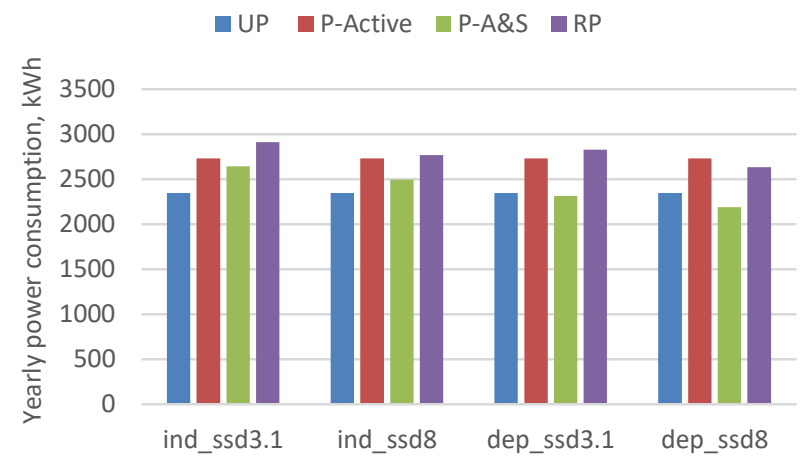

Fig. 10 Yearly power consumption of the ITS optical backhaul network

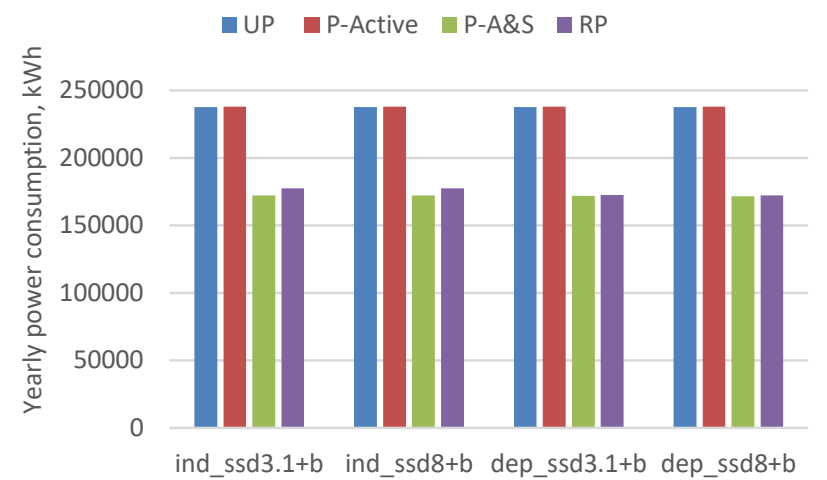

Fig. 11 Yearly power consumption of the total GPON network, including ITS optical backhaul and FTTB

Figs. 15 and 16 show the yearly power consumption for the two cases: only optical ITS backhaul (Fig. 15) and the entire GPON with the FTTB (Fig. 16). These two cases show the difference in the impact of protection on power consumption, depending on the ratio of protected elements to the unprotected.

Fig. 15 shows that yearly power consumption of the ITS backhaul only in the brownfield scenario is similar to the greenfield scenario with $\mathrm{SR}=8$ and port use of the spare ports (20\% of the FTTB), which results in six ONUs per PS. Thus, for the independent clustering there are in total seven PSs and for dependent - eight. In the brownfield case, there can be seven ports used per PS, which results in six PSs in the independent and dependent clustering. To a great extent, the number of PSs defines the number of active elements, and hence the power consumption of the brownfield scenario is less than that of the greenfield scenario with $\mathrm{SR}=8$. However, it is higher than that of the greenfield scenario with $\mathrm{SR}=16$ or 32 .

Note that the max SSD in this case is determined not only by the public transport activity pattern, but also by the FTTB users. As it was shown in [1], the maximum SSD for residential users (FTTB) is eight hours. For the brownfield scenario the maximal SSD thus is eight hours as the OLT port can be put to sleep only if all the corresponding ONUs are also in sleep mode.

Fig. 16 depicts the total power consumption by the GPON network, including that of the ITS backhaul and the buildings. We observe that in this case, the trends are similar to the results in [13]. In a hybrid optical network case, presented in [13], only the Macro Base Stations (MBSs) are protected. The majority of the ONUs, i.e., buildings, stay unprotected. This leads to a moderate increase in power consumption due to the active elements used for protection. In the optical backhaul for ITS case all the users, i.e., ONUs located at RSUs have to be protected. Thus, already the always active protection case features significantly higher power consumption compared to the unprotected case.

Generally, protection of all the ONUs results in greater sensitivity to SSD and clustering method. This sensitivity can be seen in Fig. 15, where the P-Active results in significant increase in yearly power consumption compared to the unprotected architecture. Moreover, RP scheme can result in more power consumption as P-Active due to additional active equipment, when the SSD is not adequate. In Fig. 16, there is no such obvious dependency. If we take into account the number of buildings in the total GPON network, i.e., 4877 ONUs, an increase in the power consumption due to protecting ITS ONUs (RSUs), i.e., 38 ONUs, is marginal, about $0.2 \%$ compared to the unprotected. Therefore, also for the P-A\&S and RP there is no significant difference in power consumption compared to each other and unprotected case.

Fig. 17 summarizes the FF lengths per PS and LMF lengths per ONU for working and protection paths. The FF in this case is reused from the FTTB dark fiber and is presented for completeness. The difference in total fiber length between the results of activity-independent and dependent clustering is $19 \%$. Activity-dependent clustering allows a reduction in power consumption by $3 \%$ at a cost of increased fiber length by $11 \%$.

Finally, connection availability of the optical ITS backhaul for the brownfield scenario is illustrated in Fig. 18. We observe that, as in the greenfield scenario, protection increases the connection availability to four nines independent of clustering, i.e., fiber length. Furthermore, P- 
A\&S and RP with dependent clustering allow power consumption reduction.

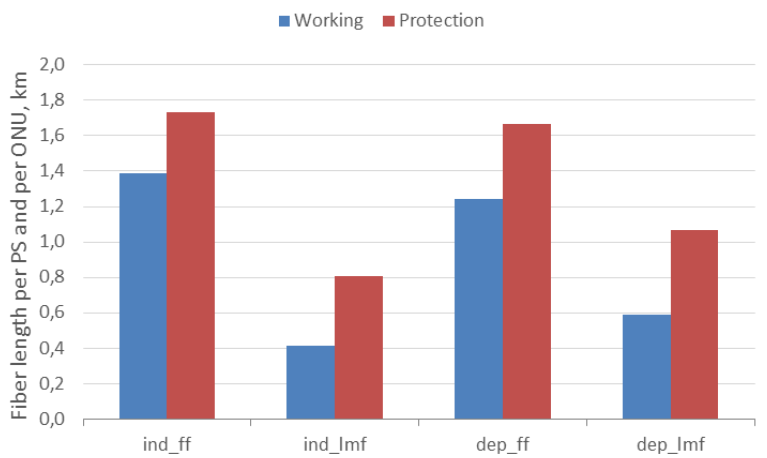

Fig. 12 Fiber lengths for working and protection FF per PS and LMF per ONU (optical ITS backhaul)

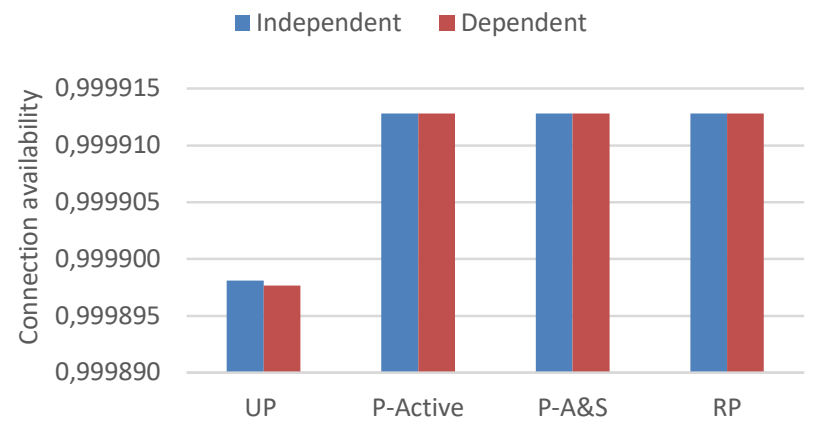

Fig. 13 Connection availability of the brownfield scenario

The choice of the protection scheme depends on the foreseen scenario and its metrics. For public ITS, the two most important deployment criteria are connection availability and failure detection time. From this perspective, in both greenfield and brownfield scenarios, the $\mathrm{RP}$ scheme is the most suitable due to its low failure detection time, see Table III in Greenfield scenario.

\section{CONCLUSION}

This paper compares three survivable architectures to protect optical access networks. The architectures differ mainly on the failure detection mechanism. The comparison is based on the evaluation of the power consumption, the failure detection time, the required fiber length as well as connection availability. The three architectures are compared to the unprotected solution and applied to a realistic ITS case study in which the required DSRC RSUs need to be interconnected with a centralized Service Server. The study compares a greenfield and a brownfield deployment scenario and investigates the impact of different splitting ratios, user grouping approaches, and Sleep Slot Durations to the performance of the considered architectures. Through detailed comparisons, the results show that the Reflective Disjoint Fiber Protection architecture offers the most appropriate solution for ITS deployment. This architecture provides fast failure detection even in sleep mode-enabled settings whilst maintaining a low yearly power consumption and providing more than four nines connection availability.

\section{ACKNOWLEDGEMENT}

This work is supported by Go8-DAAD Joint Research Cooperation Scheme. This work has been funded by the
German Research Foundation (DFG) under grant numbers MA6529/2-1 and KE1863/4-1.

\section{REFERENCES}

[1] R. Urata, C. Lam, H. Liu and C. Johnson, "High Performance, Low Cost, Colorless ONU for WDMPON," in Optical Fiber Communication (OFC) Conference, Washington DC, USA, 2012.

[2] C. Lange, R. Hülsermann, D. Kosiankowski, F. Geilhardt and A. Gladisch, "Effects of network node consolidation in optical access and aggregation networks on costs and power consumption," in Proc. SPIE 7621, Optical Metro Networks and Short-Haul Systems II, 2010.

[3] M. Forzati, A. Bianchi, J. Chen, K. Grobe, B. Lannoo, C. Mas Machuca, J. Point, B. Skubic, S. Verbrugge, E. Weis, L. Wosinska and D. Breuer, "Next Generation Optical Access Seamless Evolution," Journal of Optical Communications and Networking, vol. 7, 2015.

[4] M. Rahman, C. Mas Machuca, K. Grobe and W. Kellerer, "Advantages of Joint Access Network Planning in Dense Populated Areas," in 19th European Conference on Network and Optical Communications, NOC, Milan, Italy, 2014.

[5] A. Shahid, C. Mas Machuca, L. Wosinska and J. Chen, "Comparative Analysis of Protection Schemes for Fixed Mobile Converged Access Networks based on Hybrid PON," in IEEE Conference of Telecommunication, Media and Internet Techno-Economic (CTTE), Munich, Germany, 2015.

[6] E. Wong, C. Mas Machuca and L. Wosinska, "Survivable Hybrid Passive Optical Converged Network (HPCAN) Architectures Based on Reflective Monitoring," IEEE/OSA J. Lightw. Technol., vol. 34, no. 18, pp. 4317-4328, 2016.

[7] K. Dar, M. Bakhouya, J. Gaber, M. Wack and P. Lorenz, "Wireless communication technologies for ITS applications [Topics in Automotive Networking]," IEEE Communications Magazine, pp. 156-162, 2010.

[8] Z. Filali and F. Hameed Mir, "LTE and IEEE 802.11p for vehicular networking: a performance evaluation," EURASIP Journal on Wireless Communications, vol. http://jwcn.eurasipjournals.com/content/2014/1/89, 2014.

[9] J. Harding, G. R. Powell, R. Yoon, J. Fikentscher, C. Doyle, D. Sade, M. Lukuc, J. Simons and J. Wang, "Vehicle-to-vehicle communications: Readiness of V2V technology for application.," (Report No. DOT HS 812 014)., Washington, DC, 2014.

[10] R. Bera, J. Bera, S. Sil, S. Dogra, N. B. Sinha and D. Mondal, "Dedicated short range communications (DSRC) for intelligent transport system," in IFIP International Conference on Wireless and Optical Communications Networks, Bangalore, India, 2006.

[11] E. Wong, M. Mueller, P. Dias, C. Chan and M. Amann, "Energy-efficiency of optical network units with vertical-cavity surface-emitting lasers," Optics Express, vol. 21, pp. 14960-14970, 2013.

[12] ITU-T G.775, "Loss of Signal (LOS), Alarm Indication Signal (AIS) and Remote Defect Indication (RDI) defect detection and clearance criteria for PDH signals," ITU, 
1998.

[13] C. Mas Machuca, E. Wong, M. Furdek and L. Wosinska, "Energy Consumption and Reliability Performance of Survivable Hybrid Passive Optical Converged Networks," in Advanced Photonics 2016 (IPR, NOMA, Sensors, Networks, SPPCom, SOF), OSA Technical Digest (online) (Optical Society of America, 2016), paper NeTu3C.2..

[14] U.S. Department of Transportation's John A. Volpe National Transportation Systems, "Status of the Dedicated Short-Range Communications Technology and Applications," 2015.

[15] E. Grigoreva, C. Mas Machuca and W. Kellerer, "Optical backhaul network planning for DSRC-based Public Intelligent Transportation System: A case study," in 8th International Conference on Transparent Optical Networks (ICTON), Trento, Italy, 2016.

[16] J. Gozalvez, M. Sepulcre and R. Bauza, "IEEE 802.11p Vehicle to Infrastructure Communications in Urban Environments," IEEE Communications Magazine, pp. 176-183, 2012.

[17] Tarifverbund, Münchner Verkehrs- und Tarifverbund, [Online]. Available: http://www.mvv-muenchen.de.

[18] Open Street Map, 2016. [Online]. Available: http://www.openstreetmap.org/.

[19] ESRI 2016. ArcGIS Desktop: Release 10.3.1. Redlands, CA: Environmental Systems Research Institute, [Online].

[20] J. W. Sons, Introduction to Reliability Engineering, New York: Wiley, 1987.

[21] E. Wong and K. Lee, "Characterization of highlysensitive and fast-responding monitoring module for extended-reach passive optical networks," Optics Express, vol. 28, pp. 9019-9030, 2012.

[22] S. McGettrick, F. Slyne, N. Kitsuwan, D. Payne and M. Ruffini, "Experimental End-to-End Demonstration of Shared N:1 Dual Homed Protection in SDN-controlled Long Reach PON and Pan-European Core," IEEE/OSA J. Lightwav. Technol, vol. 34, no. 18, pp. 4205 - 4213, 2016.

[23] E. Wong, C. Mas Machuca and L. Wosinska, "Survivable Hybrid Passive Optical Converged Network (HPCAN) Architectures Based on Reflective Monitoring," , vol. 34, no. 18, pp. 4317-4328, 2016," IEEE/OSA J. Lightw. Technol., vol. 34, no. 18, pp. 4317 4328, 2016.

[24] Münchner Verkehrs- und Tarifverbund, "Journey planner: Timetable Book," 2016. [Online]. Available: http://efa.mvv-muenchen.de/index.html\#trip@enquiry. [Accessed 0902 2017].

[25] H. H. Lee, K. O. Kim, J. H. Lee and S. S. Lee, "Power saved OLT and ONU with cyclic sleep mode operating in WDM-PON," in International Conference on ICT Convergence (ICTC), 2012.

[26] M. Vogt, R. Martens and T. Andvaag, "Availability modeling of services in IP networks," in Fourth International Workshop on Design of Reliable Communication Networks, 2003. 